review

\title{
Ionising radiation and trans-generational instability
}

\author{
Ivana Vrhovac, Goran Nikšić \\ Faculty of Science University of Zagreb, Croatia
}

\begin{abstract}
Background. Indirect monitoring of the impact posed by ionising radiation to the genome instability of the descendants, consequent to the irradiation of one of their parents, boils down to the investigation of changes occurring exclusively in the mini-satellite loci of the cells constituting the gametal developmental line. The resultant mini-satellite mutations are expressed in their percentages, and equal to the ratio of the number of mutated alleles in that particular generation over the total number of alleles present. The impact of ionising radiation to the irradiated parent's offspring was first noticed on haematopoietic mouse stem-cells. Even though an irradiated cell of a female parent lacks any mutations whatsoever, daughter cells present with the increased mutation rates. The observed phenomenon of the so called trans-generational instability has been defined as the occurrence of mutations in the genome of individuals originating from the irradiated ancestors. Due to the aforementioned, one can conclude that these mutations need not be present in the irradiated parental cells, and do not necessarily vanish in the next few generations, but may result in the increase in mutation rates observed in the latter.
\end{abstract}

Conclusions. The results of the investigations performed on the animal model, as well as of those carried out in human population, point to the occurrence of significant changes to be found on mini-satellite loci of the descending generation, while the mechanism underlying those changes hasn't been completely clarified yet, and, therefore, calls for the further investigation.

Key words: radiation ionizing; radiation effects; microsatellite repeats

\section{Introduction}

It has been well-recognised that ionising radiation is capable of inducing structural changes of biologically essential macromolecules, resulting in the development

Received 28 August 2007

Accepted 10 September 2007

Correspondence to: Ivana Vrhovac and Goran Nikšić, Faculty of Science University of Zagreb, Rooseveltov trg 6, 10000 Zagreb, Croatia; Phone +385 14877 700; E-mail: ivanavrhovac@yahoo.com E-mail: marsonia2258@yahoo.com of malignancy within a human organism. ${ }^{1}$ Therefore, the development and perfecting of biomonitoring methods which enable the follow-up of early indicators of cellular alterations caused by irradiation (i.e. the indicators of the reactions exhibited by irradiated cells), dependent on the radiation type and dose delivered, are critical. Last years have witnessed a significant advancement in the detection of effects of parental irradiation, apparent in their offspring, i.e. the indirect effect of irradiation expressed as the genome instability of their descendents. ${ }^{2-6}$ 
Monitoring of the irradiation impact carried throughout a few generations subsequent to the irradiation of one of the parents, actually boils down to the investigation of changes encountered in the mini-satellite loci of the referent population, the latter being narrowed exclusively to the cells constituting the gametal developmental line..$^{7-10}$ The parameter employed in the follow-up of their frequency, the so called mutation rate, is calculated as the number of mutated alleles present in the referent generation, over the total number of alleles hosted therein, and expressed either in form of ratio, or percentage of the mutations encountered.

The impact of ionising radiation on the offspring of the first parental (F1), or the next few generations stemming from the irradiated individual, was first noticed on stem haematopoietic mouse cells. Although an irradiated cell of the female parent lacked any mutations whatsoever, the increase in the mutation rate of the daughter cells had been noticed. The phenomenon was termed the trans-generational instability, and defined as an uncommon and frequent occurrence of mutations to be found in the genome of individuals stemming from irradiated ancestors (parents, grandparents). Nevertheless, those mutations need not be present in the irradiated ancestors' cells, and do not necessarily vanish in the next generation, however, may lead to the increase in mutation rate observed in some of the descendants embraced by the next few generations. ${ }^{11}$

The results of experimental studies conducted on mice, and the results of the investigation carried out in persons irradiated in Chernobyl, have pointed to significant changes of mini-satellites hosted by the descendants, even though, as communicated by Dubrova affiliated with the Genetic Department of the Leicester University (United Kingdom), many issues haven't been resolved yet. ${ }^{12-14}$

\section{DNA satellites}

DNA satellites (micro- and mini-) are considered to be frequently-repeated segments of the cellular genome. Within this frame, micro-satellites represent shorter repeats, measuring about $500 \mathrm{pb}$ in their total length, with the basic repeat of 1-4 pb.

Mini-satellites encountered in the human genome, are spots where frequent homologous recombination takes place, located adjacent to the telomere sequences that do not undergo transcription. However, there exist also mini-satellites present in noncoded inter-genetic genome sequences, i.e. the sequences that, following transcription, represent parts of the intron. Mini-satellites are more complex, and are constituted of repeats measuring $10-100 \mathrm{pb}$ in size, and $0.1-20 \mathrm{~kb}$ in length. Most of the repeats host the GGGCAGGAXG pattern, within which an $X$ can be any nucleotide whatsoever. The most frequently encountered mini-satellites are polyA and polyT, as well as CT/AG and $\mathrm{CA} / \mathrm{TG}$ sequences, greatly resembling the chi sequence of E. coli, which represents a recombinant signal. Mini-satellites encountered in human genome are spots where homologous recombination takes place more frequently (the recombinant "hot spots"). However, as stated already, there exist also mini-satellites present in noncoded inter-genomic sequences, as well as the ones prone to exprimation, such as the MUCI locus, which contains the gene for the hyper-variable glycoprotein recovered from body fluids and some tissues, constituted of frequently-repeated mini-satellite sequences. ${ }^{14}$

Satellite DNAs are prone to mutations. Mutations of mini-satellites encountered in humans, comprise complex intra-allele rearrangements and alterations of the sequence length (mostly expansion). Minisatellite mutations encountered in human populations, may arise spontaneously only 
in cells constituting the gametal developmental line, and are characterised by the high, $0.5-13 \%$-mutation frequency per gamete, while mutations encountered in micro-satellite sequences may be found in somatic cells as well.

Most of the mini-satellite loci (90\%), are situated in the sub-telomere chromosomal regions, and had been considered irrelevant for quite a long time. They were believed to be the parts of the genome usually called "the junk DNA", although nowadays an issue of their potential influence on the alternative splicing, genome imprinting signal and regulation of gene expression, established on the transcriptional level, has been raised. ${ }^{8,15-17}$

Differences in length of satellite sequences can be easily detected with gel electrophoresis subsequent to the polymerase chain reaction (PCR). The starters (probes) employed with the polymerase chain reaction, are complementary to the parts of the genome surrounding the repeats. Their hyper-variability within population, and easiness of their detection, were the exact reasons for their forensic implementation. Based on the comparison of length of a certain number of standard satellite loci, recovered from samples taken from a number of persons, positive identification can be made. ${ }^{18,19}$ The exact determination of the loci and their mutations is accomplished by the hybridising Southern blotting method. ${ }^{20}$

\section{Trans-generational instability observed with irradiated mice}

The first experimental model utilised in the investigation of trans-generational instability, which made use of mini-satellite monitoring, was represented by the mice ESTR loci. Namely, the mini-satellites present in mice markedly differ from those present in humans, and are divided into two groups: real mini-satellites, measuring $0.5-10 \mathrm{~kb}$ in length, and having 14-17 bp long repeated patterns, and simple tandem repeated sequences, called the ESTR (expanded simple tandem repeats), measuring $0.5-16 \mathrm{~kb}$ in length, and having 4-6 bp long repeats. ${ }^{12,13,15,21}$ Mice ESTR loci contain shorter repeated patterns, and their mutations occur both in somatic and gametal developmental line cells. Though in some features (length of the repeated sequence, high content of $\mathrm{G} / \mathrm{C}$ base pairs, and locus length) resembling more those found in humans, mice mini-satellites do not exhibit mutations of a complex intra-allele-arranging type, and do not present with a high mutation rate that can be run across in human population. ${ }^{22-25}$

One of the most significant results in this research area is that of the experiment conducted in male mice of the CBA/

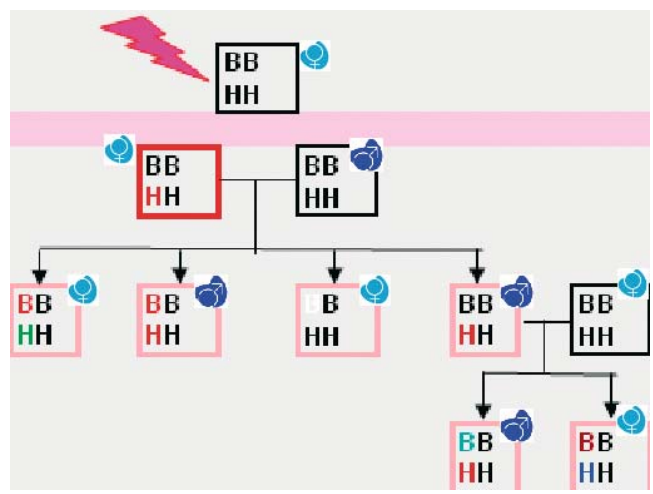

Figure 1. Model describing the inheritance of mutations in transgenic instability. Studied independent alleles are represented by the letters $B$ and $H$, wild type is written in black and mutant types are written in various colours. Each colour represents a different kind of mutation. The genotype of the irradiated mouse is in the red box, of his direct offspring is in the pink boxes and in the black boxes is the genotype of non irradiated individuals. The picture shows that the mechanism of the inheritance doesn't obey the rules of Mendelian inheritance and that mutations in minisatellite alleles that weren't mutated in parents can occur. Epigenetic mechanism is the best explanation for this type of inheritance. 
$\mathrm{H}$ strain, irradiated with various radiation types (acute X-ray irradiation, chronic gamma-irradiation, and fission high-LET neutrons). ${ }^{12,13}$ At post-irradiation week 3, 6 and 10, irradiated males had copulated with non-irradiated females of the same strain. It has been held true that a time-period elapsed from the irradiation time-point, is of importance, since male germinative cells had stemmed from irradiated post-meiotic spermatozoids (at post-irradiation week 3), pre-meiotic spermatogonia (at post-irradiation week 6) and stem cells (at post-irradiation week 10) (Figure 1). Results obtained with all radiation types employed, had demonstrated statistically significant rise in the number of mutations emerging from the ESTR loci of F1 and F2 mice generation. The frequency of mutations was very similar to that of parental samples, and failed to exhibit the tendency towards diminishment. Some of the research results point at the increase in mutation rates encountered among younger generations, while genome repeats point towards trans-generational instability exhibited following ionising radiation exposure of one of the parents. These research results have reflected in a significant decrease in number of mice required for radiation experiments as compared to classical biomonitoring methods, capable of detecting genome changes solely in case a few thousands or even a few hundred thousands mice have been used. The detection of statistically significant number of mutations located on the ESTR loci, called for the employment of only 300-400 mice. In line with the foregoing, the investigation of mini-satellites proved better and more sensitive tool to be engaged in the monitoring of radiation impact, than classical cytological techniques did. ${ }^{26}$

Promising results of the first experiments conducted in mice, motivated Dubrova and associates to continue their research in an attempt to disclose the mechanism under- lying the occurrence of mutations on the ESTR loci, the importance of gameto-generic phase in which the irradiation took place, the importance of mice strain involved, and the importance of the type of irradiation employed. In light of the foregoing, aside from $\mathrm{CBA} / \mathrm{H}$, also other mice strains, such as $\mathrm{C} 57 \mathrm{BL} / 6$ and $\mathrm{BALB} / \mathrm{c}$, have been used. The results of the research conducted in mice of parental generation, irradiated with low-LET X-rays and low-LET fission neutrons, were engaged for the purpose of disclosing the mechanism underlying the mutation occurrence. To this goal, changes on two independent loci of mice genome, Ms6-hm i Hm-2, have been observed. The results revealed the origin of mutated alleles (either in a non-irradiated female, or in an irradiated male parent), to be detectable in most of the animals involved. A crucial part of the experiment was represented by the determination of mutations in gametes of the descendants whose genotype came as a result of coupling of sperms developed from irradiated post-meiotic spermatozoids (at post-irradiation week 3). As concerns the genotype of the offspring in question, the initial research results failed to reveal an increase in number of mutations, which led to the conclusion that the basic mechanism involved in trans-generational instability becomes active not sooner than the diploid gametal-generic stage. However, a further investigation yielded confusing results, since the rate of mutations found in the offspring in reference, proved higher than that observed with the offspring of the control group, i.e. that originating from the nonirradiated male parent (the father). Under such terms, one is tempted to conclude that the source (the cause) of genetic instability encountered in the offspring, arises in fact either from diploid zygote following impregnation, or in the early embryo-generic stage, rather than during meiosis, as first assumed. The possibility that the source of 
instability lies within the very sperms was rejected based on substantial compactness and biochemical inactivity of their chromatin. Another surprising phenomenon encountered during the course of the study, was the occurrence of mutations located on the allele inherited from non-irradiated mother, which means that genome instability induced by the irradiation of the male parent, affects the alleles inherited from another parent as well. ${ }^{27}$

There exist two theories on the mechanism underlying trans-generational instability. According to one of them, mutation is a result of a direct damage inflicted to the DNA molecule in the area hosting one of the ESTR loci. If that is the case, in the offspring of an irradiated father (and their descendants), mutations located in the allele inherited from the father should be dominant. In oppose, mutations are equally encountered in both alleles of all descendants, ruling this possibility out. In addition, the number of changes encountered in 3-4 $\mathrm{kb}$ region, established in the population, is 100-fold greater than that statistically expected in case of a mutation arising from a direct DNA damage. ${ }^{28}$ According to the second theory, mutations are included into a certain set of genes, such as those responsible for DNA repair. A mutation of the aforementioned kind might lead to the one engaging both alleles. If that is the case, in line with Mendel's Laws of Inheritance, each generation would witness a decrease in mutation rate based on the crossover with non-irradiated females hosting wild gene types. However, in this particular case, the mutation rate is similar in individuals of all generations. Following the rejection of these theories, the cause of trans-generational instability proposed in the literature, has been an epigenetic effect of a still nonclarified background.

The experimental research has indicated that the frequency of mutations to be seen in the offspring of irradiated mice depends on the strain used in the experiments. Within this context, the BALB/c mice strain has been demonstrated to be the most sensitive one, while the C57BL/6 turned out to be the most stable one. The importance of utilisation of various mice strains lies within the corroboration of the fact that trans-generational instability is not a strainspecific phenomenon. Alike, the research into the genetic features of mice used for experimental purposes, contributes to the comprehension of mechanisms responsible for the mutations in reference. The results obtained in mice irradiated with doses of $0.4 \mathrm{~Gy}, 1 \mathrm{~Gy}$ and $2 \mathrm{~Gy}$, are of significance in proving that both low- and high-LET irradiation may induce trans-generational instability, and that the rate of mutations encountered in the offspring is linearly dose-dependent.

Alike human mini-satellites, mice ESTR sequences had been considered irrelevant and inert for a fairly long time. However, it has been proven that a number of changes encountered in the offspring of irradiated mice belonging to various strains (for instance, the increase in lung and skin tumour rate, the rate of blood cell malignancies and the decrease in ovarian cells' impregnation rate), are inherited conformant to the transgenerational instability model. ${ }^{27}$

\section{Specificity of hyper-mutable mini- satellites and the transgenic model}

The fact remains that, apart from human genome, there exists no other organism containing hyper-mutable mini-satellite loci in its genome. Therefore, the results of the investigation carried out on mice ESTR loci, could not, beyond reasonable doubt, be extrapolated to humans. Having in mind that mice mini-satellites follow the pattern similar to that in humans and resemble the 
latter in mutation rates, the potential solution to the problem would be the introduction of human mini-satellite loci into the mice genome. The transgenic individuals of such kind have been irradiated insofar; however, no such thing as the rise in rates of mutations encountered on these loci, nor the occurrence of trans-generational instability, have been noted. ${ }^{17}$

The trans-generational instability was first noticed in transgenic yeasts (Saccharomyces cerevisae), in which human mini-satellite loci had been introduced. In these models, even the protein that takes control over mini-satellite expansion that occurs during meiosis (termed the Rad 1), had been discovered. However, the instability noted in yeasts markedly differ from that in humans; for instance, mini-satellite mutations are usually boiled down to simple arrangements, lacking any trace of complex inter- and intra-allele rearrangements that can be found in people. In addition, in mutants in whom Rad 27 protein has been affected, complex post-mitotic mini-satellite mutations occur in somatic cells as well, while in people the latter are limited to the gametal developmental line. In some individuals, a sequence diminishment has been observed as well, as oppose to humans who presented with the preferred sequence expansions. ${ }^{17}$ Therefore, the sole manner of monitoring and investigating mini-satellite mutations in humans, are population studies.

\section{Population studies}

The results of the investigations indicating the presence of a post-irradiation risk imposed on as many as three mice generations launched a debate on potential risks for the human population, too. It is of note that mutations occurring on human mini-satellites are exclusively narrowed down to the cells of the gametal developmental line, and are of a more complex nature, so that mice models can not be considered relevant. Although the investigation of mini-satellite mutations occurring in humans are far more difficult to explore, due to the need of having a correspondent control group, the samples had been collected among families exposed to Chernobyl catastrophe, as well as among persons included into radioactive testing conducted in the Kazakhstan region Semipalatinsk, and cancer patients receiving radiotherapy. Due to the fact that the research in question was conducted on a relatively small number of persons exposed to different radiation types, as well as due to the impossibility of determining the exact irradiation dose delivered to the exposed persons based on the timing of their irradiation within the set timeframe, this study yielded contradictory results. Owing to the short half-life of unstable radioisotopes to which the families victimised by the Chernobyl catastrophe have been exposed, doses measurable immediately after the catastrophe were not relevant after all. Realistic doses were estimated based on the number of stable and unstable chromosomal aberrations detected in the exposed persons. ${ }^{29}$ Blood samples had been collected from the families dwelling the Mogilev County of Belarus, affected by the Chernobyl catastrophe, while British families served as controls. The mini-satellite analysis revealed the mutation rate registered in irradiated family members, to be 1.6-fold higher than that of controls. In addition, an elevated mutation rate was observed also with children coming from parents deemed as high dose recipients. However, due to the inadequacy of the controls, i.e. the fact that control families were of different nationality and observed a lifestyle different from that of the exposed group, the results of these investigations were given no credit. In view of solving this problem, an adequate control group was 
picked up, and compared to the members of the Ukrainian families (the areas under investigation were Zhitomir and Kiev Counties). For the aforementioned purpose, blood from children conceived prior and following the nuclear catastrophe had been sampled. In subjects conceived following the nuclear accident, a 60\%-rise in mutation rate had been established. In addition, the exposed persons had been subjected to the genotype analyses of the cells constituting the gametal developmental line, on the occasion of which only mutations in male cells (not in female ones) had been determined, despite the fact that both genders were exposed to equal radiation doses. ${ }^{30}$

Similar research had also been conducted among families dwelling Kazakhstan region of Semipalatinsk who had resided in the vicinity of the nuclear facility nearby which, starting from 1949 up to 1989, as many as 470 nuclear tests had been performed. The families in reference lived nearby the area of atmospheric, as well as underground and surface explosions. Persons of similar nationality, parental age, professions, and smoking habits, originating from the former Taldy Kurgan County of Kazakhstan, but not residing in the vicinity of the aforementioned location, served as controls. Alike the previous two, this research yielded positive results in terms of mutation rate elevation, in this particular case by as much as $70 \%$ as compared to the controls. In all of the aforementioned studies conducted in the territory of the former USSR, probes for the same mini-satellite alleles had been used. ${ }^{26,29}$

However, there exist also studies which failed to confirm the aforementioned results. For instance, the investigations conducted among children who managed to survive the most notorious event of an acute exposure to an enormous radiation dose, i.e. the Hiroshima bombing, failed to confirm the theory of trans-generational instability. Negative results of this study may serve as a proof that only certain types of radiation, or exposure (for instance, the Semipalatinsk families had been exposed to chronic, fairly uniform radiation, while the Hiroshima families had been exposed to an acute high-dose irradiation), are capable of inducing such a phenomenon. As a limitation of the studies conducted among children who managed to survive the Hiroshima bombing, the fact that most of the children under investigation were born 10 years after the nuclear attack on their parents, has often been put forward. This fact is most often taken as the main reason for negativity of the results of the study in question, together with the possibility that there exists a time-period after which the epigenetic mechanism of mutation induction "turns off". Negative results were also obtained with the analysis of sperm sampled from males diagnosed with seminoma both prior and following radiotherapy, who had been subjected to $0.4-0.8$ Gy radiation, delivered in 15 fractions. The reason for the negativity of the results might be the fact that less-fractionated doses do not exhibit an additive effect. Additionally, negative results were obtained with the investigations conducted among descendants of the workers who took part in cleansing of the Chernobyl facility following the nuclear accident, and were exposed to daily radiation doses of $0.25 \mathrm{~Gy}$. In confirmation of these results, the studies performed on mice managed to prove that a larger number of less fractionated doses lead to the same effect as does the single one, equal to their sum. ${ }^{30,31}$

\section{Mini-satellite diseases}

Mutations presented on mini-satellite loci are often put forward as causes of various diseases, for instance some tumours and 
certain types of diabetes mellitus, although their genetic background remains to be somewhat unclear. The investigations of this kind have focused on the mini-satellite locus HRAS1, the mutations of which are incriminated to cause or contribute to the development of various diseases, e.g. ovarian cancer, and hereditary breast and gall bladder cancer. One of the most prominent articles in the field, had been founded on the investigations of the HRAS1 locus, conducted in white females suffering from an ovarian cancer. Ovarian cancer is reported to be the fifth most common cause of death of US females, and the risk factors responsible for its more frequent onset are considered to be white race, older age, nuliparity and oral contraception. The mini-satellite HRAS1 locus encountered within general population consists of 4 typical alleles and 12 atypical rare ones, the latter being trusted to emerge from the mutations of those 4 typical ones. Following the investigation of the HRAS1 locus, performed in peripheral lymphocytes and tumour tissues of the sick women, as well as by virtue of comparison with the control samples taken from healthy white women of the similar age, a $50 \%$-rise in share of rare alleles of the HRAS1 locus had been noted. Statistical analyses had revealed a 1.66-fold rise in risk of developing the disease, encountered in women hosting one rare allele, and as much as 2.86 -fold rise in the referent risk posed to the women hosting two of such alleles. These results prove beyond doubt that HRAS1 locus plays a role in the onset of ovarian cancer, although the exact underlying mechanism remains to be unclear. It has been well-recognised that even as many as 4 nuclear transcript factors are bound to this allele, and that, under in vitro conditions, some of the alleles may pose as a transcription silencers or enhancers. ${ }^{31}$ The diseases that might arise as a consequence of trans-generational instability in- duced by ionising radiation are leukaemia and Hodgkin lymphoma. Such pathology had been observed in children born nearby the Sellafield Nuclear Plant of the United Kingdom. Namely, children dwelling this area were noted to have an extraordinarily high incidence of the diseases in question. The results of the study performed as early as in 1990, pointed to an extremely high risk of developing leukaemia and/or Hodgkin lymphoma, assessed in children whose parents had, prior to the conception, worked in the power plant in question, and had been exposed to substantial radiation doses. ${ }^{32}$

\section{Further investigation}

The investigation conducted insofar, have demonstrated that ionising radiation is capable of influencing the genome of non-irradiated descendants, more precisely their mini-satellite loci. Further investigations, which might disclose the mechanisms underlying the occurrence of mutations on mini-satellite loci and trans-generational instability, should aim at monitoring of mini-satellite mutations to be used as a biomonitoring tool engaged in the assessment of risk of developing malignancies, present in the offspring. In addition, there exists the possibility of application of their results to the goal of designing more efficient radiotherapeutic protocols and safety-atwork measures to be observed in persons dealing with ionising radiation sources. ${ }^{17}$ Significant improvement in understanding of the occurrence of micro-satellite mutations, is expected to be attained by the application of biophysical spectroscopic methods (for instance, NMR, circular dicroism), although the question why sequence expansions are preferred over deletions has been partially answered already, under the wing of the theory that this should be 
attributed to the difference in stability of alternative DNA molecular structures. ${ }^{33}$

\section{References}

1. Garaj-Vrhovac V, Kopjar N, Ražem D, Vekić B, Miljanić S, Ranogajec-Komor M. Application of the alkaline comet assay in biodosimetry: assessment of in vivo DNA damage in human peripheral leukocites after $\gamma$-radiation incident. Radiat Prot Dosim 2002; 98: 407-16.

2. Neel JV, Schull WJ, Awa AA, Satoh C, Kato H, Otake $\mathrm{M}$, et al. The children of parents exposed to atomic bombs, estimates of the genetic doubling dose of radiation for humans. Am J Hum Genet 1990; 46: 1053-72.

3. Sankaranarayanan K. Ionizing radiation and genetic risks X. The potential "disease phenotypes" of radiation-induced genetic damage in humans, perspectives from human molecular biology and radiation genetics. Mutat Res 1999; 429: 45-83.

4. Kodaira M, Satoh C, Hiyama K, Toyama K. Lack of effects of atomic-bomb radiation on genetic instability of tandem-repetitive elements in human germ-cells. Am J Hum Genet 1995; 57: 1275-83.

5. Dubrova YE, Nesterov VN, Krouchinsky NG, Ostapenko VA, Neumann R, Neil DL, et al. Human minisatellite mutation rate after the Chernobyl accident. Nature 1996; 380: 683-6.

6. Byrne J, Rasmussen SA, Steinhorn SC, Connelly RR, Myers MH, Lynch CF, et al. Genetic diseases in offspring of long-term survivors of childhood and adolescent cancer. Am J Hum Genet 1998; 62: 45-52.

7. Kelly R, Bulfield G, Collick A, Gibbs M, Jeffreys AJ. Characterization of a highly unstable mouse minisatellite locus: Evidence for somatic mutation during early development. Genomics 1989; 17: 1218.

8. Dubrova YE, Jeffreys AJ, Malashenko AM. Mouse minisatellite mutations induced by ionizing radiation. Nature Genet 1993; 5: 92-4.

9. Sankaranarayanan K, Chakraborty R. Ionizing radiation and genetic risks $\mathrm{XI}$. The doubling dose estimates from the mid-1950s to present and the conceptual changes to the use of human data on spontaneous mutation rates and mouse data on induced mutation rates for doubling dose calculations. Mutat Res 2000; 453: 107-27.
10. Vergnaud G, Denoeud F. Minisatellites, mutability and genome architecture. Genome Res 2000; 10: 899-907.

11. Little JB. Radiation carcinogenesis. Carcinogenesis 2000; 21: 397-404.

12. Dubrova YE, Plumb M, Brown J, Fennely J, Bois P, Goodhead D, et al. Stage specificity, dose response and doubling dose for mouse minisatellite germline mutation induced by acute radiation. Proc Natl Acad Sci 1998; 95: 6251-5.

13. Dubrova YE, Plumb M, Gutierrez B, Boulton E, Jeffreys AJ. Transgenerational mutation by radiation. Nature 2000; 405: 37.

14. Strachan T, Read A. Human Molecular Genetics 2. Oxford: BIOS Scientific Publishers Ltd; 1999.

15. Sadamoto S, Suzuki S, Kamiya K, Kominami R, Dohi K, Niwa O. Radiation induction of germline mutation at a hypervariable mouse minisatellite locus. Int J Radiat Biol 1994; 65: 549-57.

16. Bois P, Stead JHD, Bakshi S, Williamson J, Neumann R, Moghadaszadeh B, et al. Isolation and characterization of mouse minisatellites. Genomics 1998; 50: 317-30.

17. Bois PR. Hypermutable minisatellites, a human affair. Genomics 2003; 81: 349-55.

18. Armour JAL, Brinkworth $\mathrm{MH}$, Kamischke A. Direct analysis by small-pool PCR of MS205 minisatellite mutation rates in sperm after mutagenic therapies. Mutat Res 1999; 445: 73-80.

19. May CA, Tamaki K, Neumann R, Wilson G, Zagars G, Pollack A, et al. Minisatellite mutation frequency in human sperm following radiotherapy. Mutat Res 2000; 453: 67-75.

20. Bruce A, Roberts K, Lewis J, Raff M, Walter P, Johnson A. Molecular biology of the cell ( $4^{\text {th }}$ edition): New York: Garland Pub; 2002.

21. Fan YJ, Wang Z, Sadamoto S, Ninomiya Y, Kotomura N, Kamiya K, et al. Dose-response of radiation induction of a germline mutation at a hypervariable mouse minisatellite locus. Int J Radait Biol 1995; 68: 177-83.

22. Jeffreys AJ, Tamaki K, MacLeod A, Monckton DG, Neil DL, Armour JAL. Complex gene conversion events in germline mutation at human minisatellite. Nature Genet 1994; 6: 136-45.

23. Jeffreys AJ, Neumann R. Somatic mutation process at a human minisatellite. Hum Mol Genet 1997; 6: 129-36. 
24. Tamaki K, May CA, Dubrova YE, Jeffreys AJ. Extremly complex repeat shuffling during germline mutation at human minisatellite B6.7. Hum Mol Genet 1999; 8: 879-88.

25. Buard J, Collick AJ, Brown J, Jeffreys AJ. Somatic versus germline mutataion process at minisatellite CEBI (D2S90) in human and transgenic mice. Genomics 2000; 65: 95-103.

26. Dubrova YE. Transgenerational germline instability. 33th Annual Meeting of the European Environmental Mutagen Society" Aberdeen, Great Britain 2003, Abstract pp 18.

27. Barber R, Plumb MA, Boulton E, Roux I, Dubrova YE. Elevated mutation rates in the germ line of first- and second-generation offspring of irradiated male mice. Proc Natl Acad Sci USA 2002; 99: 687782.

28. Niwa O, Kominami R. Untargeted mutation of the maternally derived mouse hypervariable minisatellite allele in F1 mice born to irradiated spermatozoa. Proc Natl Acad Sci USA 2002; 98: 1705-10.

29. Dubrova YE. Monitoring of radiation-induced germline mutation in human. Swiss Medical Weekly 2003; 133: 474-8.

30. Dubrova YE, Grant G, Chumal AA, Stezhka VA, Karakasian AN. Elevated minisatellite mutation rate in the post chernobyl families from Ukraine. Am J Hum Genet 2002; 71: 801-9.

31. Jeffreys N, Weitzel JN, Ding S, Larson GP, Nelson RA, Goodman A, et al. The HRASI minisatellite locus and risk of ovarian cancer. Cancer Res 2000; 60: 259-61.

32. Gardner MJ, Snee MP, Hall AJ, Powell CA, Downes S, Terrell JD. Results of case-control study of leukaemia and lymphoma among young people near Sellefield nuclear plant in West Cumbria. Br Med J 1990; 300: 423-9.

33. Wells RD, Dere R, Hebert ML, Napierala M, Son LS. Advance in mechanisms of genetic instability related to hereditary neurological diseases. $\mathrm{Nucl}$ Acids Res 2005; 33: 3785-798. 